\title{
Obstructive Sleep Apnea Is Closely Related to Cardiovascular Risk Factors, but Not to Clinical Recurrence of Atrial Fibrillation after Catheter Ablation: An Analysis of Atrial Fibrillation Patients
}

\author{
Chan Joo Lee ${ }^{a}$ Tae-Hoon Kim ${ }^{b}$ Sungha Park ${ }^{b}$ Hui-Nam Pak ${ }^{b}$ \\ aDepartment of Health Promotion, Severance Hospital, Yonsei University College of Medicine, Seoul, \\ Republic of Korea; ${ }^{\mathrm{b}}$ Division of Cardiology, Severance Cardiovascular Hospital and Cardiovascular \\ Research Institute, Yonsei University College of Medicine, Seoul, Republic of Korea
}

\section{Keywords}

Cardiovascular risk · Obstructive sleep apnea · Atrial fibrillation recurrence

\begin{abstract}
Background: Obstructive sleep apnea (OSA) is a well-known predictor of atrial fibrillation (AF). However, OSA usually accompanies other risk factors of AF. We tried to investigate whether OSA is related to AF recurrence after catheter ablation. Methods: A total of 378 patients (mean age $59.9 \pm 10.7$ years, $72.5 \%$ male) who underwent catheter ablation of AF were enrolled and underwent overnight ambulatory polysomnography before the ablation procedure. These patients were examined once every 3 months at the outpatient clinic to determine AF recurrence. Results: Based on the apnea-hypopnea index $(\mathrm{AHI})$, we divided the study subjects into 3 groups defined as mild $(\mathrm{AHI}<10)$, moderate $(10<\mathrm{AHI}<30)$, or severe $(\mathrm{AHI}>30)$ OSA. Patients with severe OSA had a higher prevalence of hypertension, diabetes mellitus, and coronary artery disease (CAD). However, AF recurrence was not different between the three groups. The Kaplan-Meier analysis also showed no significant difference in AF recurrence according to the degree of severity of OSA. Multivariate logistic regression analysis revealed that OSA might be a predictor of CAD; however, Cox regression analysis showed that only early recurrence is closely related to AF recurrence after catheter ablation, rather than the severity of OSA. Conclusion: This study shows that the severity of OSA is not associated with the recurrence of AF after catheter ablation in Korean patients. Treatment of OSA for the sole indication of lowering AF recurrence may need to be reconsidered.
\end{abstract}


Lee et al.: The Irrelevance of OSA to AF Recurrence

\section{Introduction}

Obstructive sleep apnea (OSA) is a chronic disorder characterized by repeated cessation of air flow during sleep despite respiratory efforts [1]. It is relatively common with a prevalence of $2 \sim 7 \%$ in the general population [2]. OSA causes various physiologic changes such as sympathetic activation, endothelial dysfunction, vascular oxidative stress, and inflammation, which are all related to cardiovascular disease including atrial fibrillation (AF) [3]. Recent studies showed that continuous positive airway pressure (CPAP) therapy for OSA raises the efficacy of radiofrequency catheter ablation (RFCA) in patients with severe OSA [4-6]. However, these studies should be interpreted with caution because their study populations were relatively small and their diagnosis of OSA was insufficient. In addition, patients with OSA usually have associated other cardiovascular risk factors. Therefore, in this study, we investigated the sole impact of OSA on the recurrence of AF after RFCA in Korean patients with AF.

\section{Methods}

\section{Study Population}

All patients gave informed consent for inclusion in the Yonsei AF Ablation Cohort Database. This study population consisted of 378 patients with AF who underwent RFCA at our institution from December 2011 through May 2014. The study exclusion criteria were as follows: (1) permanent AF, refractory to electrical cardioversion, (2) AF with valvular disease $\geq$ grade 2, (3) associated structural heart disease other than left ventricular hypertrophy, (4) a history of prior RFCA or cardiac surgery, and (5) patients who did not have sleep apnea assessments. In order to visualize the anatomy of the pulmonary vein (PV) and the left atrium (LA), 3D-cardiac CT images were acquired in all patients. All antiarrhythmic drugs were discontinued for a minimum period of 5 half-lives, and amiodarone was stopped at least 4 weeks before the procedure. This study received prior approval from the Institutional Review Board at the Yonsei University Health System (IRB No. 4-2014-1080).

\section{Diagnosis of OSA}

Before catheter ablation, polysomnography was performed with a portable device (Embletta X-100; Embla Systems Inc., Broomfield, CO, USA), which is a level 2 sleep monitoring system, according to the recommendations of the European Respiratory Society and the European Society of Hypertension [7]. None of the patients used CPAP at baseline. The device recorded data including the electroencephalogram, electromyogram, electrocardiogram (ECG) or heart rate, airflow, respiratory effort, and oxygen saturation. Polysomnographic data were manually scored by an experienced specialist in accordance with the definition of OSA syndrome by the American Academy of Sleep Medicine [8]. Apnea was defined as a $>90 \%$ decrease in airflow for $>10 \mathrm{~s}$ in the presence of thoracoabdominal ventilatory efforts, and hypopnea was defined as a $>50 \%$ reduction in airflow with desaturation of $>3 \%$ or arousal for $>10 \mathrm{~s}$, or $>30 \%$ reduction in airflow with desaturation of $>4 \%$ for $>10 \mathrm{~s}$ in the presence of thoracoabdominal ventilatory efforts. The apnea-hypopnea index (AHI) was calculated by dividing the total duration of apnea and hypopnea by the total sleep time. Patients were grouped according to OSA severity, which was classified as previously described [4]: mild OSA $(\mathrm{AHI}<10)$, moderate OSA $(10 \leq \mathrm{AHI}<30)$, severe OSA (AHI $\geq 30)$. Desaturation was defined as an oxygen saturation of $\leq 90 \%$, and the desaturation index was defined as the number of desaturation episodes per hour of sleep. The Pittsburgh Sleep Quality Index (SQI) and the Epworth Sleepiness Scale (ESS) data were collected from participants before polysomnography.

\section{Electrophysiologic Mapping and RFCA}

The details of the electrophysiologic mapping and RFCA technique and strategy were as described in previous studies $[9,10]$. Briefly, RF energy was delivered using an open irrigated-tip catheter (Celsius, Johnson and Johnson Inc., Diamond Bar, CA, USA; Coolflex, St. Jude Medical Inc., Minnetonka, MN, USA; $30-$ $35 \mathrm{~W} ; 47^{\circ} \mathrm{C}$ ). All patients initially underwent circumferential PV isolation and bidirectional block of the cavotricuspid isthmus. For the patients with persistent AF, we added a roof line, a posterior inferior line, and an anterior line as the standard lesion set. The operator could choose to perform additional ablations in the 
superior vena cava or non-PV foci, or conduct complex fractionated electrograms at his discretion. The procedure was complete when there was no immediate recurrence of AF after cardioversion with isoproterenol infusion ( $5 \mu \mathrm{g} / \mathrm{min}$ ). If there were mappable AF triggers or atrial premature beats, we carefully mapped and ablated those non-PV foci as much as possible. All RFCA procedures were conducted by 2 operators with over 10 years of experience, and according to the specific protocol listed above.

Follow-Up after Ablation

All patients were given no anti-arrhythmic drugs after RFCA. Patients were asked to visit follow-up appointments at 1, 3, 6, 9, and 12 months after RFCA, and every 6 months thereafter in an outpatient clinic. An ECG was obtained at every visit, and additional ECGs were recorded when patients reported symptoms suggestive of AF. A Holter ECG ( 24 or $48 \mathrm{~h}$ ) and/or an event recorder were worn at 3, 6, 12, 18, and 24 months after RFCA according to the 2012 HRS/EHRA/ECAS Expert Consensus Statement guidelines [11]. Recurrence of AF was defined as any episode of AF or atrial tachycardia lasting longer than $30 \mathrm{~s}$. AF episodes within the 3-month blanking period during follow-up were regarded as an early recurrence. Any ECG documentation of AF recurrence after 3 months was diagnosed as clinical recurrence, which was analyzed as endpoint in this study.

Statistical Analysis

Continuous variables were expressed as mean \pm SD and were compared using a one-way analysis of variance (ANOVA) or an independent $t$ test. Categorical variables were summarized as numbers and percentages of the group total. Pearson's $\chi^{2}$ test was performed to compare categorical variables. The association between the severity of OSA and coronary artery disease (CAD) was rated using multiple logistic regression analysis. AF recurrence-free survival curves for each group were presented as Kaplan-Meier plots and compared by log-rank test. Univariate and multivariate Cox regression analyses were conducted to identify the predictor of AF recurrence. All statistical analyses were performed using SPSS version 20.0 for Windows (Statistical Package for the Social Sciences Inc., Chicago, IL, USA). A $p$ value of $<0.05$ was considered statistically significant.

\section{Results}

OSA as a Risk Factor for CAD in Patients with AF

Table 1 shows baseline characteristics of the study participants according to the severity of their OSA. Overall, the patients were middle aged ( $59.9 \pm 10.7$ years old), overweight (body mass index [BMI] 25.1 \pm 3 ), and had normal cardiac function (left ventricular ejection fraction $63 \pm 7.9 \%$ ). Anthropometric data revealed that weight and BMI had a tendency to be higher in the moderate and severe OSA groups; however, this trend did not reach statistical significance. Notably, neck diameter and waist circumference were significantly higher in the severe OSA group compared to the mild OSA group. Patients with moderate and severe OSA were older and had higher incidences of diabetes mellitus (DM) and hypertension than those with mild OSA. Although left ventricular function was not different among the groups, LA volume was larger in the moderate and severe OSA groups. Notably, the prevalence of CAD was significantly higher with increasing severity of OSA $(p<0.001)$.

In order to evaluate the role of OSA as a predictor of CAD, multivariate logistic regression analysis was performed (online suppl. Table 1; for all online suppl. material, see www. karger.com/doi/10.1159/000489854). This analysis revealed that severe OSA was a powerful risk factor for CAD, even though it was not statistically significant in the regression model adjusted for both DM and hypertension as a confounding factor.

Relationship between OSA and the Recurrence of AF

After ablation of AF, the mean duration of the follow-up period was $20.2 \pm 9.7$ months, and the time to clinical recurrence was $11.8 \pm 8.1$ months. With respect to the presence or 
Lee et al.: The Irrelevance of OSA to AF Recurrence

Table 1. Clinical characteristics according to the severity of OSA

\begin{tabular}{|c|c|c|c|c|}
\hline & $\begin{array}{l}\text { Mild OSA } \\
(n=190)\end{array}$ & $\begin{array}{l}\text { Moderate OSA } \\
(n=131)\end{array}$ & $\begin{array}{l}\text { Severe OSA } \\
(n=57)\end{array}$ & $p$ value \\
\hline Age, years & $55.7 \pm 11.5$ & $61.7 \pm 8.9$ & $63 \pm 8.8$ & $<0.001$ \\
\hline Male & $123(64.7)$ & $107(81.7)$ & $44(77.2)$ & 0.006 \\
\hline Type of AF & & & & 0.542 \\
\hline Persistent & $69(36.3)$ & $50(38.2)$ & $17(29.8)$ & \\
\hline Paroxysmal & $121(63.7)$ & $81(61.8)$ & $40(70.2)$ & \\
\hline Hypertension & $70(36.8)$ & $80(61.1)$ & $38(66.7)$ & $<0.001$ \\
\hline DM & $23(12.1)$ & $20(15.3)$ & $14(24.6)$ & 0.029 \\
\hline Prior stoke or TIA & $24(12.6)$ & $24(18.3)$ & $11(19.3)$ & 0.131 \\
\hline CHA2DS2-VASc & $1.5 \pm 1.3$ & $2 \pm 1.5$ & $2.3 \pm 1.7$ & $<0.001$ \\
\hline Coronary artery disease & $15(7.9)$ & $26(19.8)$ & $16(28.1)$ & $<0.001$ \\
\hline Valvular heart disease & $18(9.5)$ & $10(7.6)$ & $3(5.3)$ & 0.292 \\
\hline Systolic blood pressure, $\mathrm{mm} \mathrm{Hg}$ & $118.5 \pm 14.8$ & $119.0 \pm 10.7$ & $122.1 \pm 13.1$ & 0.581 \\
\hline Diastolic blood pressure, $\mathrm{mm} \mathrm{Hg}$ & $75.4 \pm 10.8$ & $73.5 \pm 9.3$ & $75.7 \pm 5.4$ & 0.725 \\
\hline LA dimension, $\mathrm{mm}$ & $41 \pm 5.8$ & $42.7 \pm 5.6$ & $42.9 \pm 7.2$ & 0.016 \\
\hline LA volume index, $\mathrm{mL} / \mathrm{m}^{2}$ & $30.8 \pm 15$ & $34.9 \pm 14.6$ & $34.2 \pm 15.7$ & 0.038 \\
\hline LVEF, \% & $63.4 \pm 7$ & $62.5 \pm 8.2$ & $63 \pm 9.9$ & 0.606 \\
\hline $\mathrm{E} / \mathrm{E}^{\prime}$ & $10.5 \pm 4.5$ & $10.8 \pm 4.7$ & $10.3 \pm 4$ & 0.75 \\
\hline LV mass index, $\mathrm{g} / \mathrm{m}^{2}$ & $68.9 \pm 42.4$ & $75.3 \pm 43$ & $75.1 \pm 47.3$ & 0.372 \\
\hline LVEDD, mm & $49.4 \pm 4.4$ & $49.8 \pm 4.3$ & $50.1 \pm 5.6$ & 0.596 \\
\hline LVESD, mm & $33.4 \pm 5.7$ & $33.6 \pm 5.6$ & $34.1 \pm 6.7$ & 0.772 \\
\hline Height, cm & $166.2 \pm 9.1$ & $164.7 \pm 16.4$ & $168 \pm 9.2$ & 0.219 \\
\hline Weight, kg & $68.7 \pm 11.8$ & $69.8 \pm 9.9$ & $72.6 \pm 12.5$ & 0.072 \\
\hline BMI & $24.8 \pm 3.1$ & $25.3 \pm 2.6$ & $25.6 \pm 3.2$ & 0.093 \\
\hline Neck diameter, $\mathrm{cm}$ & $36.8 \pm 3.2$ & $37.5 \pm 2.6$ & $38.1 \pm 3.2$ & 0.008 \\
\hline Waist circumference, $\mathrm{cm}$ & $89.3 \pm 11.2$ & $91.6 \pm 8.9$ & $95.3 \pm 8.5$ & $<0.001$ \\
\hline hsCRP, mg/dL & $3 \pm 8.3$ & $1.6 \pm 2.9$ & $2 \pm 2.5$ & 0.138 \\
\hline AHI, apnea + hypopnea/h & $3.8 \pm 3$ & $17.8 \pm 5.7$ & $44.5 \pm 13$ & $<0.001$ \\
\hline ESS & $6.8 \pm 4.1$ & $6.8 \pm 4.6$ & $6.3 \pm 4$ & 0.675 \\
\hline SQI & $5.2 \pm 3.1$ & $4.9 \pm 3$ & $5.5 \pm 3.5$ & 0.393 \\
\hline Test time, min & $406.8 \pm 89.4$ & $437.6 \pm 86.9$ & $435.3 \pm 96.2$ & 0.005 \\
\hline Mean duration of OSA, s & $11.3 \pm 9.6$ & $21 \pm 6.8$ & $24.9 \pm 6.2$ & $<0.001$ \\
\hline Mean duration of hypopnea, s & $20.1 \pm 7.2$ & $23.6 \pm 4.9$ & $24.7 \pm 5.5$ & $<0.001$ \\
\hline Minimum oxygen saturation, $\%$ & $89.7 \pm 3.6$ & $85.2 \pm 4.3$ & $81.5 \pm 4.4$ & $<0.001$ \\
\hline Desaturation index, event/h & $3.4 \pm 2.8$ & $15.5 \pm 6.1$ & $39.3 \pm 14$ & $<0.001$ \\
\hline Early recurrence & $58(30.5)$ & $42(32.1)$ & $14(24.6)$ & 0.581 \\
\hline Antiarrhythmic drug after procedure & $31(16.3)$ & $27(20.6)$ & $7(12.3)$ & 0.342 \\
\hline
\end{tabular}

$p$ values $<0.05$ are marked in bold. Data are presented as $n(\%)$ or mean \pm SD. AHI, apnea-hypopnea index; $\mathrm{AF}$, atrial fibrillation; BMI, body mass index; DM, diabetes mellitus; ESS, Epworth sleepiness scale; hsCRP, high sensitive C reactive protein; LA, left atrium; LV, left ventricle; LVEDD, LV end diastolic dimension; LVEF, LV ejection fraction; LVESD, LV end systolic dimension; OSA, obstructive sleep apnea; SQI, sleep quality index; TIA, transient ischemic attack.

absence of clinical recurrence, LA dimension, type of AF, and early recurrence were significantly different between each group (Table 2). The use of antiarrhythmic drug after catheter ablation was more frequent in patients with clinical recurrence. The average times to recurrence were $11.3 \pm 7.4$ months in the mild OSA group, $13.3 \pm 9.3$ months in the moderate OSA group, and $7.9 \pm 3.4$ months in the severe OSA group. However, clinical recurrence rate and duration were not statistically different between the groups. There was no difference in the use of antiarrhythmic drug according to the severity of OSA (Table 1). 
Lee et al.: The Irrelevance of OSA to AF Recurrence

Table 2. Clinical characteristics according to the recurrence of atrial fibrillation

\begin{tabular}{|c|c|c|c|}
\hline & $\begin{array}{l}\text { No recurrence } \\
(n=313)\end{array}$ & $\begin{array}{l}\text { Recurrence } \\
(n=65)\end{array}$ & $p$ value \\
\hline Age, years & $58.7 \pm 10.8$ & $59.8 \pm 10.7$ & 0.443 \\
\hline Male & $223(71)$ & $52(80)$ & 0.14 \\
\hline Type of AF & & & 0.006 \\
\hline Persistent & $103(32.8)$ & $33(50.8)$ & \\
\hline Paroxysmal & $211(67.2)$ & $32(49.2)$ & \\
\hline Hypertension & $156(49.7)$ & $33(50.8)$ & 0.873 \\
\hline DM & $46(14.6)$ & $11(16.9)$ & 0.641 \\
\hline Prior stoke or TIA & $49(15.6)$ & $10(15.4)$ & 0.964 \\
\hline CHA2DS2-VASc & $1.8 \pm 1.5$ & $1.7 \pm 1.4$ & 0.817 \\
\hline Coronary artery disease & $49(15.6)$ & $8(12.3)$ & 0.498 \\
\hline Valvular heart disease & $25(8)$ & $6(9.2)$ & 0.734 \\
\hline Systolic blood pressure, $\mathrm{mm} \mathrm{Hg}$ & $119.2 \pm 14.8$ & $118.4 \pm 9.3$ & 0.772 \\
\hline Diastolic blood pressure, $\mathrm{mm} \mathrm{Hg}$ & $74.7 \pm 10.8$ & $75.5 \pm 7.7$ & 0.743 \\
\hline LA dimension, $\mathrm{mm}$ & $41.6 \pm 6$ & $43.2 \pm 5.8$ & 0.046 \\
\hline LA volume index, $\mathrm{mL} / \mathrm{m}^{2}$ & $32.3 \pm 15.1$ & $34.3 \pm 15.5$ & 0.323 \\
\hline LVEF, \% & $63.1 \pm 7.9$ & $62.4 \pm 7.9$ & 0.512 \\
\hline $\mathrm{E} / \mathrm{E}^{\prime}$ & $10.6 \pm 4.4$ & $10.4 \pm 4.8$ & 0.812 \\
\hline $\mathrm{LV}$ mass index, $\mathrm{g} / \mathrm{m}^{2}$ & $72 \pm 43.8$ & $72.4 \pm 41.4$ & 0.95 \\
\hline LVEDD, mm & $49.5 \pm 4.6$ & $50.1 \pm 4.4$ & 0.334 \\
\hline LVESD, mm & $33.5 \pm 6$ & $34 \pm 4.6$ & 0.482 \\
\hline Height, cm & $166.4 \pm 8.8$ & $163.9 \pm 22.2$ & 0.132 \\
\hline Weight, kg & $69.6 \pm 11.3$ & $69.7 \pm 11.8$ & 0.951 \\
\hline BMI & $25.1 \pm 3$ & $25 \pm 3.1$ & 0.965 \\
\hline Neck diameter, $\mathrm{cm}$ & $37.2 \pm 3.1$ & $37.4 \pm 2.6$ & 0.651 \\
\hline Waist circumference, $\mathrm{cm}$ & $1 \pm 0.7$ & $1 \pm 0.5$ & 0.576 \\
\hline hsCRP, mg/dL & $2.5 \pm 6.7$ & $1.6 \pm 1.8$ & 0.052 \\
\hline AHI, apnea + hypopnea/h & $15.0 \pm 16.0$ & $13.6 \pm 12.6$ & 0.508 \\
\hline ESS & $6.6 \pm 4.2$ & $7.2 \pm 4.4$ & 0.508 \\
\hline SQI & $5.1 \pm 3.2$ & $5.3 \pm 2.6$ & 0.348 \\
\hline Test time, min & $420.6 \pm 92.6$ & $427 \pm 79.7$ & 0.602 \\
\hline Mean duration of OSA, s & $16.8 \pm 9.8$ & $15.9 \pm 10.9$ & 0.520 \\
\hline Mean duration of hypopnea, s & $21.9 \pm 6.5$ & $22.1 \pm 6.7$ & 0.827 \\
\hline Minimum oxygen saturation, $\%$ & $86.6 \pm 5.1$ & $88.2 \pm 4$ & 0.01 \\
\hline Desaturation index & $13.2 \pm 14.7$ & $12.1 \pm 10.8$ & 0.568 \\
\hline Early recurrence & $81(25.8)$ & $33(50.8)$ & $<0.001$ \\
\hline Antiarrhythmic drug after procedure & $28(8.9)$ & $13(20.3)$ & $<0.001$ \\
\hline
\end{tabular}

$p$ values $<0.05$ are marked in bold. Data are presented as $n(\%)$ or mean \pm SD. Abbreviations are explained in Table 1.

Table 3 shows the results of the Cox regression analysis for risk factors of the recurrence of $\mathrm{AF}$. In the univariate model, LA diameter, persistent AF, and early recurrence were significantly related to the clinical recurrence of AF; however, the severity of OSA was not associated with clinical recurrence. Multivariate Cox regression analysis revealed that only early recurrence was a significant risk factor for clinical recurrence after adjustment for other confounding factors. However, the severity of OSA was not a predictor of clinical recurrence (Table 3; Fig. 1). 
Table 3. Univariate and multivariate Cox regression analyses for the recurrence of atrial fibrillation

\begin{tabular}{|c|c|c|c|c|}
\hline \multirow[t]{2}{*}{ Predictor } & \multicolumn{2}{|l|}{ Univariate } & \multicolumn{2}{|l|}{ Multivariate } \\
\hline & hazard ratio & $p$ value & hazard ratio & $p$ value \\
\hline Age & $1.01(0.98-1.03)$ & 0.541 & $1.00(0.98-1.03)$ & 0.722 \\
\hline Male gender & $1.53(0.83-2.8)$ & 0.173 & $1.50(0.8-2.84)$ & 0.210 \\
\hline LA diameter & $1.04(1-1.09)$ & 0.037 & 1.02 (0.97-1.07) & 0.445 \\
\hline LVEF & $0.99(0.96-1.02)$ & 0.361 & $0.99(0.96-1.02)$ & 0.448 \\
\hline Hypertension & $1.03(0.63-1.68)$ & 0.899 & $0.95(0.55-1.64)$ & 0.865 \\
\hline $\mathrm{DM}$ & $1.13(0.59-2.15)$ & 0.722 & $1.02(0.5-2.07)$ & 0.962 \\
\hline BMI & $1.00(0.92-1.09)$ & 0.962 & $1.00(0.91-1.1)$ & 0.920 \\
\hline Persistent AF & $1.85(1.14-3.01)$ & 0.013 & $1.39(0.79-2.43)$ & 0.254 \\
\hline Early recurrence & $2.71(1.66-4.41)$ & $<0.001$ & $2.63(1.59-4.37)$ & $<0.001$ \\
\hline ESS & $1.04(0.98-1.1)$ & 0.232 & $\mathrm{NI}$ & NI \\
\hline SQI & $1.02(0.95-1.1)$ & 0.585 & $\mathrm{NI}$ & $\mathrm{NI}$ \\
\hline AHI & $1.00(0.98-1.01)$ & 0.739 & NI & NI \\
\hline Mean duration of OSA & $0.99(0.97-1.02)$ & 0.553 & NI & NI \\
\hline Mean duration of hypopnea & $1.00(0.96-1.04)$ & 0.924 & NI & NI \\
\hline Desaturation index & $1.00(0.98-1.02)$ & 0.815 & NI & NI \\
\hline Mild OSA & - & - & - & - \\
\hline Moderate OSA & $1.39(0.83-2.33)$ & 0.216 & $1.08(0.61-1.91)$ & 0.785 \\
\hline Severe OSA & $0.83(0.36-1.88)$ & 0.649 & $0.73(0.31-1.73)$ & 0.477 \\
\hline
\end{tabular}

$p$ values $<0.05$ are marked in bold. Abbreviations are explained in Table 1. NI, not included.

Fig. 1. Kaplan-Meier curve for AFfree survival after catheter ablation according to the severity of OSA.

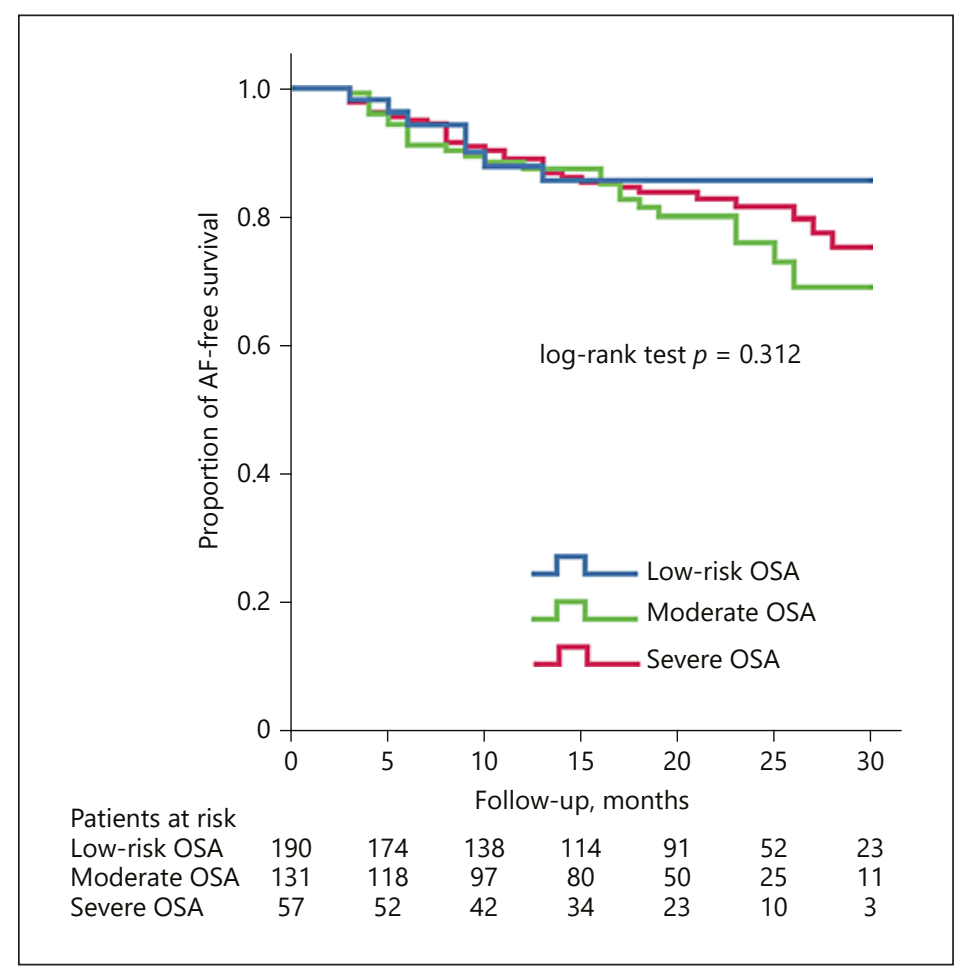


Lee et al.: The Irrelevance of OSA to AF Recurrence

\section{Discussion}

The findings of this study are as follows: (1) OSA is closely related with cardiovascular disease and/or risk factors such as age, DM, and abdominal obesity among the patients with $\mathrm{AF}$; (2) however, the severity and the existence of OSA are not predictors of the recurrence of $\mathrm{AF}$ after ablation; (3) after adjustment of possible confounding factors, early recurrence of AF is the only predictor of the clinical success of ablation for the treatment of AF.

Hypertension is well known to be related with sleep apnea [12]. According to previous research, it appears that $50 \%$ of patients with OSA are hypertensive and $30 \%$ of hypertensive patients have OSA $[13,14]$. Furthermore, patients with moderate or severe OSA have a 3 -fold elevated risk of CAD compared to patients without OSA, and it has been reported that CPAP reduces the risk of CAD in patients with OSA $[15,16]$. Our data are partially in agreement with these previous studies. The proportion of DM and hypertension in patients with severe OSA is nearly 2 -fold compared to those in patients with mild OSA. Moreover, the prevalence of CAD is higher with increased severity of OSA. Interestingly, logistic regression analysis showed that severe OSA was no longer significantly associated with CAD after adjustment for both DM and hypertension, which are major risk factors for CAD. This is probably because severe OSA patients were more likely to have DM or hypertension. Therefore, we conclude that, when studying OSA, the interplay between OSA and other clinical factors should be considered carefully.

The close relationship between OSA and AF has been widely studied and supported for a long time $[4-6,17]$. However, our data demonstrate that the severity of OSA cannot be a predictor of the recurrence of AF after catheter ablation. There are various possible reasons for the discrepancy between the results of our study and other studies. One reason may be the different amounts of energy used for AF ablation, leading to different ablation efficacies. The more energy gets delivered to the tissue, the better the outcome of the treatment [18]. According to our study protocol, we used $35 \mathrm{~W}$, which is a relatively high power. Another possibility for the clinical recurrence is that the LA diameter, which is the major substrate for development of AF, did not differ depending on the severity of OSA. The recurrence of AF after catheter ablation may occur because the electrical activity of AF was not blocked sufficiently by the procedure. The probability of recurrence was significantly higher in patients with larger LA size [19]. Furthermore, our study was conducted in the Korean population which has lower BMI even at a similar rate of hypertension, DM, and dyslipidemia compared to the western countries [20]. Our study population had lower BMI compared to the study by Fein et al. [5] and by Matiello et al. [4], which included patients with severe OSA but relatively low BMI. These demographic differences may be an important factor for the contrast between our results and other studies supporting the relationship between OSA and the recurrence of AF.

Nevertheless, there have been studies indicating that OSA is not related to AF. Mehra et al. [21] showed that OSA was closely associated with ventricular ectopy but not AF after adjustment of confounding factors, although they did not evaluate the recurrence of AF. Our study also adjusted for other cardiovascular risk factors coexisting with OSA, and the severity of OSA was not related to clinical recurrence. Chilukuri et al. [22] showed that obesity, rather than OSA, is an independent predictor of procedural failure after catheter ablation of AF. In addition, according to Tang et al. [23], the severity of OSA is not associated with the recurrence of AF. However, in those studies, OSA was diagnosed using the Berlin questionnaire, which is a major limitation. Our study used portable polysomnography, which has proven to be an effective tool for the diagnosis of breathing disorders during sleep [24].

The present study has some limitations. First, because this is an observational study, the heterogeneity of the individual OSA groups might be problematic. Second, the results may not be similar to those of other studies due to ethnic differences. Third, portable polysomnography 
was used for evaluating the severity of OSA, and it was performed only one time at enrollment. Therefore, the diagnosis and classification of OSA may lack accuracy to some degree. This is because portable polysomnography is likely to overestimate the severity of OSA in patients with a mild sleep disorder and to underestimate AHI in those with more severe OSA, compared to in-lab polysomnography $[25,26]$. Fourth, variables not included in this study might serve as confounding factors. For example, the duration of $\mathrm{AF}$ is known to be an independent predictor of maintenance of sinus rhythm [27], but was not included in this analysis.

In conclusion, this study shows that the severity of OSA is closely related with well-known cardiovascular risk factors and CAD, but it is not a predictor of the recurrence of AF after catheter ablation in Korean patients. Therefore, the relationship between OSA and AF should be further investigated under well-defined conditions. Treatment of OSA for the sole purpose of lowering AF recurrence may need to be reconsidered.

\section{Acknowledgements}

This work was supported by a grant from the Korean Health Technology R\&D Project, Ministry of Health and Welfare, Republic of Korea (HI13C0715), a grant (A085136) from the Korea Health 21 R\&D Project, Ministry of Health and Welfare, and from the National Research Foundation of Korea (NRF) funded by the Ministry of Science, ICT, and Future Planning (MSIP) (7-2013-0362).

\section{Disclosure Statement}

The authors have nothing to declare.

\section{References}

1 Sankri-Tarbichi AG: Obstructive sleep apnea-hypopnea syndrome: etiology and diagnosis. Avicenna J Med 2012;2:3-8.

2 Punjabi NM: The epidemiology of adult obstructive sleep apnea. Proc Am Thorac Soc 2008;5:136-143.

3 Shamsuzzaman AS, Gersh BJ, Somers VK: Obstructive sleep apnea: implications for cardiac and vascular disease. JAMA 2003;290:1906-1914.

4 Matiello M, Nadal M, Tamborero D, Berruezo A, Montserrat J, Embid C, Rios J, Villacastin J, Brugada J, Mont L: Low efficacy of atrial fibrillation ablation in severe obstructive sleep apnoea patients. Europace 2010;12: 1084-1089.

5 Fein AS, Shvilkin A, Shah D, et al: Treatment of obstructive sleep apnea reduces the risk of atrial fibrillation recurrence after catheter ablation. J Am Coll Cardiol 2013;62:300-305.

6 Naruse Y, Tada H, Satoh M, et al: Concomitant obstructive sleep apnea increases the recurrence of atrial fibrillation following radiofrequency catheter ablation of atrial fibrillation: clinical impact of continuous positive airway pressure therapy. Heart Rhythm 2013;10:331-337.

7 Parati G, Lombardi C, Hedner J, et al: Recommendations for the management of patients with obstructive sleep apnoea and hypertension. Eur Respir J 2013;41:523-538.

8 Epstein LJ, Kristo D, Strollo PJ Jr, et al: Clinical guideline for the evaluation, management and long-term care of obstructive sleep apnea in adults. J Clin Sleep Med 2009;5:263-276.

9 Park JH, Pak HN, Choi EJ, Jang JK, Kim SK, Choi DH, Choi JI, Hwang C, Kim YH: The relationship between endocardial voltage and regional volume in electroanatomical remodeled left atria in patients with atrial fibrillation: comparison of three-dimensional computed tomographic images and voltage mapping. J Cardiovasc Electrophysiol 2009;20:1349-1356.

10 Shim J, Joung B, Park JH, Uhm JS, Lee MH, Pak HN: Long duration of radiofrequency energy delivery is an independent predictor of clinical recurrence after catheter ablation of atrial fibrillation: over 500 cases experience. Int J Cardiol 2013;167:2667-2672.

11 Calkins H, Kuck KH, Cappato R, et al: 2012 HRS/EHRA/ECAS expert consensus statement on catheter and surgical ablation of atrial fibrillation: recommendations for patient selection, procedural techniques, patient management and follow-up, definitions, endpoints, and research trial design. Europace 2012;14:528-606.

12 Peppard PE, Young T, Palta M, Skatrud J: Prospective study of the association between sleep-disordered breathing and hypertension. N Engl J Med 2000;342:1378-1384. 
Lee et al.: The Irrelevance of OSA to AF Recurrence

13 Worsnop CJ, Naughton MT, Barter CE, Morgan TO, Anderson AI, Pierce RJ: The prevalence of obstructive sleep apnea in hypertensives. Am J Respir Crit Care Med 1998;157:111-115.

14 Duran-Cantolla J, Aizpuru F, Martinez-Null C, Barbe-Illa F: Obstructive sleep apnea/hypopnea and systemic hypertension. Sleep Med Rev 2009;13:323-331.

15 Peker Y, Carlson J, Hedner J: Increased incidence of coronary artery disease in sleep apnoea: a long-term follow-up. Eur Respir J 2006;28:596-602.

16 Marin JM, Carrizo SJ, Vicente E, Agusti AG: Long-term cardiovascular outcomes in men with obstructive sleep apnoea-hypopnoea with or without treatment with continuous positive airway pressure: an observational study. Lancet 2005;365:1046-1053.

17 Kanagala R, Murali NS, Friedman PA, Ammash NM, Gersh BJ, Ballman KV, Shamsuzzaman AS, Somers VK: Obstructive sleep apnea and the recurrence of atrial fibrillation. Circulation 2003;107:2589-2594.

18 Yuyun MF, Stafford PJ, Sandilands AJ, Samani NJ, Andre Ng G: The impact of power output during percutaneous catheter radiofrequency ablation for atrial fibrillation on efficacy and safety outcomes: a systematic review. J Cardiovasc Electrophysiol 2013;24:1216-1223.

19 Abecasis J, Dourado R, Ferreira A, Saraiva C, Cavaco D, Santos KR, Morgado FB, Adragao P, Silva A: Left atrial volume calculated by multi-detector computed tomography may predict successful pulmonary vein isolation in catheter ablation of atrial fibrillation. Europace 2009;11:1289-1294.

20 Kim Y, Suh YK, Choi H: BMI and metabolic disorders in South Korean adults: 1998 Korea National Health and Nutrition Survey. Obes Res 2004;12:445-453.

21 Mehra R, Stone KL, Varosy PD, Hoffman AR, Marcus GM, Blackwell T, Ibrahim OA, Salem R, Redline S: Nocturnal arrhythmias across a spectrum of obstructive and central sleep-disordered breathing in older men: outcomes of sleep disorders in older men (MrOS sleep) study. Arch Intern Med 2009;169:1147-1155.

22 Chilukuri K, Dalal D, Gadrey S, et al: A prospective study evaluating the role of obesity and obstructive sleep apnea for outcomes after catheter ablation of atrial fibrillation. J Cardiovasc Electrophysiol 2010;21:521-525.

23 Tang RB, Dong JZ, Liu XP, et al: Obstructive sleep apnoea risk profile and the risk of recurrence of atrial fibrillation after catheter ablation. Europace 2009;11:100-105.

24 Bruyneel M, Ninane V: Unattended home-based polysomnography for sleep disordered breathing: current concepts and perspectives. Sleep Med Rev 2014;18:341-347.

25 Iber C, Redline S, Kaplan Gilpin AM, Quan SF, Zhang L, Gottlieb DJ, Rapoport D, Resnick HE, Sanders M, Smith P: Polysomnography performed in the unattended home versus the attended laboratory setting - Sleep Heart Health Study methodology. Sleep 2004;27:536-540.

26 Bruyneel M, Sanida C, Art G, Libert W, Cuvelier L, Paesmans M, Sergysels R, Ninane V: Sleep efficiency during sleep studies: results of a prospective study comparing home-based and in-hospital polysomnography. J Sleep Res 2011;20:201-206.

27 Frick M, Frykman V, Jensen-Urstad M, Ostergren J, Rosenqvist M: Factors predicting success rate and recurrence of atrial fibrillation after first electrical cardioversion in patients with persistent atrial fibrillation. Clin Cardiol 2001;24:238-244. 